\title{
Stimmen zur aktuellen Auflage
}

„This great book offers a comprehensive and practice-oriented introduction to the variety of strategic perspectives.“

Prof. Dr. Bob de Wit, Professor für Strategic Leadership, Nyenrode Business University und Founding Director von Strategy Works, Rotterdam

„Unternehmensführung verantwortet das Ganze, sagt man. Dieses Buch liefert darüber hinaus Antworten zur steigenden Komplexität und Dynamik.“

Prof. assoc. Dr. Christian Abegglen, Gründungsdirektor der St. Galler Business School (SGBS)

„Startups und etablierte Unternehmen verbindet mehr, als oft angenommen wird. Müller \& Wrobel vermitteln anschaulich und mit vielen Beispielen Chancen, die daraus entstehen.“

Dominic Blank, Gründer und CEO POSpulse, Agile Consumer Response, Berlin

„Das Buch ist im besten Sinne praxisorientiert - mit jeder Menge theoretischem Tiefgang. Keine leichte Kost, aber dennoch gut bekömmlich und mit sehr viel Nährstoffen. Unbedingt empfehlenswert.“

Prof. Dr. Frank Habermann, Professur für ABWL, Hochschule für Wirtschaft und Recht Berlin

„In einer kompakten Form erfährt der Leser sehr nachvollziehbar und mit vielen aktuellen Fällen versehen in einer interessant verfassten Form etwas zu den wichtigsten Themen der Unternehmensführung. Dieses Konzept hat sich seit der Erstauflage bewährt.“

Prof. em. Dr. Günter Müller-Stewens, Institut für Betriebswirtschaft, Universität St. Gallen

„Dieses Buch nimmt sich Paradoxien an, denen die Unternehmensführung im Zeitalter von Digitalisierung und Wandel gegenübersteht. Dem Leser werden wissenschaftlich fundiert Lösungswege aufgezeigt, die von höchster praktischer Relevanz sind. Ein Standardwerk, das gleich mehrfach Widersprüche überwindet.“ Prof. Dr. Heike M. Hölzner, Professorin für Entrepreneurship und Mittelstandsmanagement, Hochschule für Technik und Wirtschaft Berlin

„Ein innovatives Standardwerk der Unternehmensführung, das klärt, aufklärt und nie missioniert.“

Dr. Herbert Schober-Ehmer, Redmont Consulting Cluster, Wien

„Das Buch verknüpft Theorie und Praxis auf einzigartige Weise und liefert zahlreiche illustrierende Fallbeispiele.“ 
„Endlich eine rundherum überzeugende Einführung in das große Thema Unternehmensführung. Sehr lesenswert für alle, die bei der Unternehmensführung ,state of the art' sein wollen!“

Prof. Dr. Ralf T. Kreutzer, Professor für Marketing, Hochschule für Wirtschaft und Recht Berlin

„Strategische Beidhändigkeit (Ambidextrie) wird im Zeitalter der Digitalisierung, von Corona und Klimawandel immer relevanter. Dieses Standardwerk für moderne Unternehmensführung bietet das nötige Handwerkszeug und einen Kompass für die Herausforderungen von heute.“

Dr. Stefan Groß-Selbeck, Global Managing Partner BCG Digital Ventures, Berlin

„Ein hervorragender und praxisnaher Gesamtüberblick zum Thema Unternehmensführung.“ 